\title{
Professor Dr. Herbert Döring 80 Jahre
}

Am 10. 2. 1991 begeht H. Döring seinen 80. Geburtstag. Seine auch nach der nun schon über 12 Jahre zurückliegenden Emeritierung ungebrochene wissenschaftliche Aktivität setzt eine lange Forscherlaufbahn fort, die durch wesentliche Beiträge auf dem Gebiet der Hochfrequenztechnik zu hoher internationaler Anerkennung geführt hat.

Herbert Döring wurde 1911 in Wien geboren. Er studierte Elektrotechnik an der Technischen Hochschule Wien, gleichzeitig parallel Musik an der Wiener Staatsakademie. Durch Professor Reithoffer früh für die Funktechnik begeistert, setzte er seine wissenschaftliche Ausbildung im Fernmeldetechnischen Institut von Professor Petritsch in Wien mit einer Dissertation über ,Laufzeiteffekte in Dioden bei Ultrakurzwellen" fort, die 1936 zur Promotion zum Dr. techn. führte.

Röhrentechnik und insbesondere Laufzeiteffekte bei hohen Frequenzen bilden danach einen roten Faden seines Lebens. Seine Industrietätigkeit begann 1936 bei Siemens \& Halske in Wien mit der Entwicklung von Rundfunkgeräten und kleinen Sendern. Er wechselte 1938 als wissenschaftlicher Mitarbeiter zu Professor Ramsauer in das AEG-Forschungszentrum Berlin, wo er mit dem Aufbau und der Untersuchung effizienter Triftröhren betraut wurde, und 1941 zur C. Lorenz AG, Berlin, wo ex einen größeren Bereich der Senderöhrenentwicklung übernahm und maßgeblich an der Entwicklung von Klystrons und Wanderfeldröhren für die neu erschlossene Mikrowellentechnik beteiligt war. Bei dem Wiederaufbau des Röhrenwerkes 1946 in Eßlingen/Neckar wurde er für die gesamte Entwicklung und Produktionstechnik verantwortlich sowie ab 1948 für ein neues, auf seine Initiative zurückgehendes Mikrowellen-Laboratorium, in dem die erforderliche Meß- und Schaltungstechnik bearbeitet wurde.

1949 habilitierte er sich an der Technischen Hochschule Stuttgart mit einer Arbeit über ,Neue Berechnungsverfahren zur Elektronenbewegung in Triftröhren" und hielt Vorlesungen über Ultrakurzwellenröhren und Höchstfrequenztechnik. 1952 erhielt er den Ruf als Ordinarius für Hochfrequenztechnik an die RWTH Aachen. Herbert Döring hat diesen Bereich
28 Jahre bis zu seiner Emeritierung 1980 geleitet und das Image des Institutes entscheidend mitgeprägt. Über 1500 Diplom- und Studienarbeiten entstanden unter seiner Leitung, und mehr als 80 Doktoranden, 8 alleine nach der Emeritierung, zeugen von seiner wissenschaftlichen Ausstrahlung. Etwa ein Dutzend seiner Schüler sind wiederum Ordinarien an Universitäten und Technischen Hochschulen. Das Spektrum der Forschung erweiterte sich auf Halbleiter, Laser und integrierte Mikrowellenschaltungen.

Herbert Döring war zweimal Dekan (1955/56 und 1961/62), Auswahlausschuß-Mitglied der Studienstiftung des Deutschen Volkes, Gutachter und VizePräsident der Deutschen Forschungsgemeinschaft. Er vertrat die Hochschule als Mitglied des NTG-Vorstandes und als NTG-Vorsitzender 1967/68. Er ist lange Jahre Mitglied des NTZ-Beirates und Schriftleiter des AEUं gewesen. Weitere Ehrenämter schließen die Tätigkeit als Kurator der Physikalisch Technischen Bundesanstalt ein sowie auf internationaler Ebene den stellvertretenden Vorsitz der Deutschen Sektion des IEEE.

Für sein Engagement und seine wissenschaftliche Leistung erfuhr er viele Ehrungen, u. a. den VDEEhrenring, die Wilhelm Exner Medaille des Österreichischen Gewerbevereins sowie die Ernennung zum Fellow des IEEE 1972. Er ist seit langem Mitglied der Rheinisch Westfälischen Akademie der Wissenschaften.

Auch nach seiner formalen Emeritierung wirkt Herbert Döring weiter für die Wissenschaft. In mehreren Arbeiten hat er die historischen Bezüge der Röhrenforschung seit Edison, Fleming u. a. dargestellt. Darüber hinaus gilt sein Interesse der Weiterentwicklung der neuen Höchstleistungsröhren mit transversaler Fokussierung oder Steuerung, dem Gyrotron und Gyrocon. Mit diesen Arbeiten hat er der Röhrenentwicklung in Deutschland in den letzten Jahren neue Impulse vermittelt.

Alle seine Schüler und Freunde wünschen Herbert Döring weiterhin Gesundheit und wissenschaftliche Erfolge.

\section{H. J. Schmitt, Aachen}

